\title{
Reflets
}

Revue ontaroise d'intervention sociale et communautaire

\section{La politique du workfare en Ontario : les groupes de femmes coincés entre la nécessité et l'État néolibéral}

\section{Jacinthe Michaud}

Volume 6, numéro 2, automne 2000

Problèmes sociaux en Ontario français

URI : https://id.erudit.org/iderudit/026315ar

DOI : https://doi.org/10.7202/026315ar

Aller au sommaire du numéro

Éditeur(s)

Reflets : Revue ontaroise d'intervention sociale et communautaire

ISSN

1203-4576 (imprimé)

1712-8498 (numérique)

Découvrir la revue

Citer cet article

Michaud, J. (2000). La politique du workfare en Ontario : les groupes de femmes coincés entre la nécessité et l'État néolibéral. Reflets, 6(2), 34-60.

https://doi.org/10.7202/026315ar

Tous droits réservés (C) Reflets : Revue ontaroise d'intervention sociale et communautaire, 2000
Ce document est protégé par la loi sur le droit d'auteur. L'utilisation des services d'Érudit (y compris la reproduction) est assujettie à sa politique d'utilisation que vous pouvez consulter en ligne.

https://apropos.erudit.org/fr/usagers/politique-dutilisation/ 


\section{La politique $d u$ workfare en Ontario $\square$ :}

\section{les groupes de femmes coincés entre la nécessité et l'État néolibéral}

Jacinthe Michaud
Études des femmes, Collège universitaire Glendon (York)

La politique du workfare en Ontario cible à la fois les assistés sociaux et les groupes de base issus du secteur communautaire à qui on demande d'accepter une part importante des placements de travail obligatoire (Cholette 1998). Le bon vouloir des organismes communautaires devient essentiel à l'implantation du programme et de ce fait, le gouvernement ontarien reconnaît implicitement l'existence de ce vaste secteur. Implicitement, parce que l'approche néolibérale du gouvernement Harris a procédé depuis 1995 au démantèlement des structures intermédiaires entre l'État et la société civile sous prétexte de se soustraire à l'influence des groupes voués à la défense des intérêts particuliers : féministes, écologistes, syndicalistes ou autres. Ce phénomène n'est pas unique à l'Ontario. Ce qu'on privilégie désormais, c'est le rapport direct à l'individu réduit à faire valoir seul ses besoins et à se défendre contre l'arbitraire (Jenson and Phillips 1996; Laurin 1999). Du côté de l'État, tout se passe comme si on cherchait à saper les canaux de communication établis entre les services publics et les groupes de base issus des mouvements sociaux grâce à des décennies d'efforts considérables. Comme si, après avoir dépensé tant d'efforts à l'établir, cette collaboration devenait soudainement ni nécessaire, ni désirable. Selon Laurin, le démantèlement des relations intermédiaires entraîne, comme conséquence, l'établissement d'un nouveau mode de régulation sociale. 
Sur cette toile de fond, un nouveau mode de régulation s'est mis en place au cours des dernières années. Il entraîne le démantèlement ou l'effondrement des institutions intermédiaires, celles qui organisent, au quotidien, les pratiques des sujets : leur vie familiale, leur travail, leurs loisirs, leurs activités politiques, religieuses, etc. Ces institutions du quotidien jouent le rôle de tampon, elles médiatisent les rapports entre les individus et les groupes à la base et les institutions qui coordonnent, centralisent les procès de la régulation à d'autres niveaux. Par conséquent, la suppression ou l'ébranlement des structures intermédiaires livrent les individus à l'influence de plus en plus directe, immédiate des instances supralocales des réseaux de contrôle économique, politique, administratif, symbolique (Laurin 1999 : 69).

"...les féministes, qui pendant longtemps avaient dépensé temps et énergie à faire valoir les mérites d'un rapport de partenariat à leurs opposants dans leur propre milieu..., se voient forcées de revenir à des formes d'action collective développées en dehors du champ discursif de la négociation politique. "
La participation avec les paliers de l'État, longuement débattue au sein des groupes de base, est aujourd'hui honnie par l'administration gouvernementale qui en contrôle pourtant l'essentiel des modalités. De leur côté, les féministes, qui pendant longtemps avaient dépensé temps et énergie à faire valoir les mérites d'un rapport de partenariat à leurs opposants dans leur propre milieu (Michaud 1992; 1997), se voient forcées de revenir à des formes d'action collective développées en dehors du champ discursif de la négociation politique. Pourtant, la logique néolibérale réserve toujours une place spécifique au secteur communautaire.

L'article qui suit propose une analyse de ce statut d'intermédiaire et de la nouvelle configuration des rapports entre les institutions gouvernementales et les groupes de base du secteur communautaire, en particulier les groupes de femmes, sujets qui soulèvent des préoccupations pour les intervenantes féministes et progressistes. Tout en demeurant sourd aux demandes des groupes, l'État néolibéral entend se décharger sur eux de la gestion de certains enjeux et de certains problèmes sociaux. L'exemple que nous décrirons est celui du travail obligatoire et de son intersection avec les approches d'intervention des groupes de femmes envers les assistées sociales : usagères des services ou travailleuses volontaires. 
" Notre objectif ici sera de démontrer que les groupes de base du secteur communautaire ne sont pas exempts d'une certaine ambiguité dans l'interprétation des besoins et des désirs de cette dernière."
Notre but est de démontrer que les enjeux sous-jacents au programme de workfare portent sur le contrôle du temps volontaire et sur l'établissement d'un tout nouveau mécanisme de contrôle social.

Dans un premier temps, il sera question de la nouvelle configuration du statut d'intermédiaire des groupes de base. Dans la seconde partie, nous nous pencherons sur le statut du temps volontaire au sein du secteur communautaire et sur le mandat que se sont donnés les groupes face à la détérioration des conditions d'existence des assistés sociaux, en particulier les femmes. La partie suivante abordera les positions des groupes de femmes vis-à-vis les placements de workfare et des motifs qui les sous-tendent, souvent liés aux images discursives à l'égard de l'assistée sociale. Notre objectif ici sera de démontrer que les groupes de base du secteur communautaire ne sont pas exempts d'une certaine ambiguiité dans l'interprétation des besoins et des désirs de cette dernière.

Les données de base de cet article proviennent principalement de 17 entrevues réalisées à l'été 1999 auprès d'intervenantes de groupes de femmes et auprès d'organismes communautaires oeuvrant essentiellement dans un contexte linguistique minoritaire et ce, à l'aide de questions semi-ouvertes. Les numéros qui apparaissent à l'intérieur du texte entendent préserver l'anonymat des répondantes des groupes concernés. Précisons de plus qu'une première série de 19 entrevues auprès de femmes francophones vivant de l'aide sociale en Ontario avait été réalisée à l'été 1998 avec une méthodologie qualitative, proche du récit de vie. Ces deux séries s'inscrivent à l'intérieur d'un projet de recherche sur le workfare et son impact sur les femmes francophones et les groupes de femmes qui travaillent avec elles.

\section{L'espace intermédiaire en question $\square$ : les rapports entre l'État et les groupes de femmes}

Le statut d'intermédiaire est attribué aux organisations qui se sont développées dans la deuxième moitié du $\mathrm{XX}^{\mathrm{e}}$ siècle alors que 
"Parler du statut d'intermédiaire lorsqu'il est question des groupes de femmes, c'est faire référence aux structures de communication et aux pratiques de représentation auprès de divers paliers institutionnels de l'État. " plusieurs d'entre elles cherchaient à se démarquer du type de représentation assuré jusque là par les partis politiques ou les syndicats. C'était l'époque des "nouveaux mouvements sociaux " et le début d'une ère où l'État reconnaissait - par un ensemble de programmes d'aide et de subventions-la légitimité des groupes voués à la défense des intérêts individuels et collectifs de catégories sociales défavorisées (Jenson and Phillips 1996; Mahon 1999 : 257). Parler du statut d'intermédiaire lorsqu'il est question des groupes de femmes, c'est faire référence aux structures de communication et aux pratiques de représentation auprès de divers paliers institutionnels de l'État. Ce statut est attribuable à toute une panoplie d'organisations, y inclus les plus avantagées sur le plan économique et social. Mais nous nous intéressons plus particulièrement aux groupes qui défendent les intérêts et les besoins de catégories sociales spécifiques, qui revendiquent l'amélioration des conditions d'existence et qui assurent la représentation de divers segments de la population tout en luttant contre des systèmes d'oppression.

La reconnaissance formelle du statut d'intermédiaire est inégale au Canada, le Québec étant sans doute la province la plus engagée dans la légitimation des groupes de base, en particulier ceux des femmes, en tant que partenaires dans l'établissement de programmes sociaux (Andrew 1998). Mais pour agir comme intermédiaire entre l'État et la société civile, point n'est besoin d'attendre cette reconnaissance formelle. Dans le cas de l'Ontario, le point tournant, nous rappelle Linda Cardinal, a été la Commission Royale d'enquête sur la situation de la femme vers la fin des années 60 (1992 : 8). À partir de cette époque, plusieurs organisations vouées à la défense et à la représentation des femmes francophones dans des domaines spécialisés (santé, éducation, travail, violence physique et sexuelle, pouvoir politique, égalité juridique et autres ) ont été créées pour intervenir aux niveaux national et provincial. Longtemps ignorés par les féministes, tant du Québec que du Canada anglais, les groupes de femmes de l'Ontario français agissent également comme intermédiaires dans la revendication des services en français, là où les femmes francophones sont mal servies par le bilinguisme (Cardinal 1992; 
"...les groupes

demeurent le véhicule privilégié par lequel, non seulement les pauvres et les assistés sociaux, mais un ensemble de catégories sociales de femmes et d'hommes peuvent espérer se faire entendre, n'ayant pas d'autre façon de parvenir à émerger dans l'espace public ou politique. "
Coderre 1995; Welch 1999). De plus, le réseautage des groupes de femmes entre eux-comme dans le domaine de la violence avec l'Action ontarienne contre la violence faite aux femmes ou au sein de groupes chapeautant plusieurs communautés d'intérêts, comme dans le cas de la Table féministe francophone de concertation provinciale fondée en décembre 1992-assure une visibilité accrue et un pouvoir de revendication plus étendu auprès des gouvernements fédéral et provincial.

Une autre caractéristique structurelle importante attachée au statut d'intermédiaire touche le temps volontaire. Les groupes de femmes, qu'ils soient de niveau provincial, régional ou local, se sont en grande partie constitués à partir de militantes ou de bénévoles dont la présence a rendu possible la réalisation de tout un ensemble de pratiques et de services ainsi que leur maintien dans la durée. Intervenantes salariées et intervenantes non-salariées partagent espace et temps de travail, ce qui contribue à complexifier la gestion des ressources humaines au sein de l'organisation. Même s'ils possèdent des ressources limitées et un budget fort modeste (Browne 1996:4), les groupes de femmes sont solidement implantés dans les communautés. Tant et si bien que la tendance actuelle en sciences sociales est de les considérer comme des créateurs d'emplois directs et indirects, faisant d'eux une force incontournable de l'économie sociale (Welch 1999).

Compte tenu de l'absence de reconnaissance étatique de l'action des groupes de femmes et des groupes de base issus du secteur communautaire, au sens où leur légitimité de partenaires serait prise en considération dans l'élaboration des politiques sociales, on pourrait se demander si, en Ontario, le statut d'intermédiaire est bel et bien une réalité. À cela, il faut tout de suite répondre que les groupes demeurent le véhicule privilégié par lequel, non seulement les pauvres et les assistés sociaux, mais un ensemble de catégories sociales de femmes et d'hommes peuvent espérer se faire entendre, n'ayant pas d'autre façon de parvenir à émerger dans l'espace public ou politique. Cependant, l'organisation structurelle du statut d'intermédiaire se modifie selon les formes de l'État. Les voies de la représentation des besoins, des désirs et de l'expérience vécue ${ }^{1}$ ainsi que les modes d'expression 
"... dès le tournant des années 80, la notion de partenariat en était venue à occuper la position dominante et la logique de la gestion des conflits avait fini par trouver son aboutissement au niveau institutionnel..." des revendications portées par les groupes restent multiples. Au cours de la période antérieure, les formes plus ou moins achevées de l'État-providence avaient fini par nous habituer à un chassé-croisé d'échanges et de concertation entre les groupes de femmes, rompus au réseautage, et les institutions publiques telles que la santé, l'éducation, les services sociaux. Après une période plus ou moins longue de refus, les groupes de femmes s'étaient institutionnalisés et formés au processus de la négociation et de la participation. Certes des tensions à l'intérieur du mouvement féministe contemporain s'étaient toujours fait sentir entre des positions de participation et de non-participation (Michaud 1992). Mais dès le tournant des années 80 , la notion de partenariat en était venue à occuper la position dominante et la logique de la gestion des conflits avait fini par trouver son aboutissement au niveau institutionnel (Lamoureux 1994; Michaud 1997). À tel point que même du côté de l'État, on avait pu constater que «du point de vue de l'institution, la négociation de ces relations constitue un bien valorisé, dans la mesure où les revendications et les luttes font l'objet de divers arrangements politico-institutionnels » (LustigierThaler, Maheu, Hamel 1998 : 176).

Le néolibéralisme a donné à de nombreux pays occidentaux l'opportunité de s'attaquer à ces modes de représentation. Mais il ne faut pas conclure trop rapidement à l'existence d'un vide qui se serait instauré suite au démantèlement des anciens réseaux d'échange et de concertation. Certes, l'imposition brutale du programme Ontario au travail permet de cibler, en premier lieu, des individus isolés, les mères seules étant les plus touchées (Laurin 1999; Dandurand et McAll 1996). Car le gouvernement n'a aucunement l'intention d'exempter les parents seuls ayant des enfants d'âge pré-scolaire (Cholette 1997; Workfare Watch 1999), malgré certaines déclarations qui avaient été faites avant le libellé de la loi (Moscovitch 1997 : 89). Ce sont les principes de l'éthique du travail inspirés par un des courants du néoconservatisme (Roche 1995; Michaud, à paraître) qui s'appliquent et les mères assistées sociales y sont soumises comme les autres ${ }^{2}$. Cette application stricte du principe de l'égalité des genres, sans égard aux conditions particulières, ne veut pas dire cependant que le système d'aide 
«Avec cette nouvelle configuration des rapports, les groupes de femmes ont moins à craindre l'effondrement des structures intermédiaires que de se voir imposer une toute autre fonction dans le modèle de régulation sociale qui se met en place. " sociale est uniforme et que ses divers textes et règlements ne font pas de distinction entre les femmes et les hommes, comme en fait foi la règle hétérosexiste qui obligeait une assistée sociale à déclarer la nature de ses relations avec un homme ${ }^{3}$. En l'absence de services de garde, les mères seules sont régulièrement confrontées au choix le plus difficile qui soit : réintégrer le marché du travail et assumer seules les charges d'entretien de leurs enfants avec rien de mieux qu'un salaire minimum ou demeurer prestataires avec la couverture sociale bien maigre que la loi leur réserve encore. À cela s'ajoute le fardeau d'avoir à composer avec des informations contradictoires de la part de leur agent d'aide sociale qui, par ailleurs, ne leur donne ni aide ni soutien d'aucune sorte (Conseil de planification sociale d'Ottawa-Carleton 1999; Workfare Watch 1999).

Bien que l'élimination des anciens réseaux de concertation augmente la vulnérabilité des pauvres et des assistés sociaux, il n'est pas si facile que cela de faire fi de l'action des groupes et des réseaux. Les pressions exercées sur le secteur communautaire pour mettre en place la restructuration des politiques sociales et les programmes de workfare démontrent que même une situation de confrontation et de conflit exige que soient préservés des rapports constants de négociation et de compromis (Jenson 1997; LustigierThaler, Maheu et Hamel 1998). Le statut d'intermédiaire demeure, mais il s'est transformé au point qu'il est impératif de s'interroger sur les nouveaux modes de représentation qui sont en train de s'établir. Avec cette nouvelle configuration des rapports, les groupes de femmes ont moins à craindre l'effondrement des structures intermédiaires que de se voir imposer une toute autre fonction dans le modèle de régulation sociale qui se met en place. Si l'espace politico-institutionnel est peu réceptif à la représentation féministe et progressiste, il semble à peu près inimaginable que l'État ontarien puisse parvenir à établir complètement sa restructuration sans faire l'effort de s'accommoder de la participation de vastes segments du secteur communautaire. Les nouvelles formes de la citoyenneté sociale sont donc loin d'être achevées. Dans la section qui suit, nous allons aborder la question du temps volontaire à partir de l'insertion des assistées au sein des groupes de femmes. Cette insertion issue de la communauté représente le deuxième pôle, 
après celui de l'État, par lequel les groupes maintiennent leur statut d'intermédiaires.

\section{Le statut du temps volontaire et la représentation de l'assistée}

Commençons d'abord par un rappel bref de quelques aspects du programme Ontario au travail. C'est à partir de l'automne 1997 que le programme de placements se met en place, avant donc que la loi ne soit promulguée en mai 1998. Tous les assistés sociaux y sont soumis à quelques exceptions près.

Seules les personnes âgées et les personnes handicapées sont exemptées de la participation au programme mais, de leur gré, elles peuvent choisir d'y prendre part. Les personnes qui offrent des soins à un membre de leur famille ayant des besoins particuliers, les bénéficiaires temporairement malades ou handicapées et les bénéficiaires en congé de maternité ou en congé parental sont temporairement exemptées du programme; les autres y travailleront jusqu'à 17 heures par semaine (Cholette 1998 : 108).

Le système prévoit le paiement de certains coûts tels les vêtements et le matériel de travail mais certaines études réalisées auprès des assistés sociaux ayant vécu l'expérience du workfare soulignent que l'information à cet effet ne circule pas toujours. (Conseil de planification sociale d'Ottawa-Carleton 1999; Workfare Watch 1999). Ensuite, ce sont les municipalités qui, désormais, sont responsables du soutien au revenu et de l'aide à l'emploi. Le programme de prestation familiale qui avant la réforme, relevait de la province, est aboli et tous les services aux familles monoparentales sont fondus au programme Ontario au travail (Cholette 1997 : 30). La contribution des entreprises n'est pas écartée mais ce sont les groupes de base du secteur communautaire 
"...le travail volontaire au Canada s'identifie plus à l'éthique du "devoir civique " qu'à une activité librement consentie. Cette constatation découle $d u$ secteur de la santé et des services sociaux où le militantisme féministe autant que le bénévolat ont fait naître de nombreuses organisations autonomes..." qui sont d'abord visés. Pour les gestionnaires du système, la rencontre entre les assistés et les groupes de base présente un caractère utilitaire. C'est une rencontre qui va de soi et c'est sur elle que doivent se développer les modes d'implantation du workfare et cela pour plusieurs raisons : d'abord parce que le secteur communautaire est très développé au Canada, comparativement à d'autres pays occidentaux tels ceux de l'hémisphère du Nord européen (Briskin et Eliason 1999), même si sa répartition sur l'ensemble du territoire est plutôt inégale. Ensuite, contrairement à d'autres pays toujours, le travail volontaire au Canada s'identifie plus à l'éthique du "devoir civique " qu'à une activité librement consentie. Cette constatation découle du secteur de la santé et des services sociaux où le militantisme féministe autant que le bénévolat ont fait naitre de nombreuses organisations autonomes (Briskin et Eliasson 1999 : XI; Feldberg et Carlsson 1999 : 353). Ainsi, il devient plus aisé de faire reposer sur les communautés des problèmes sociaux tels que la violence, l'aide aux adultes dépendants, aux personnes âgées, aux indigents, problèmes sociaux qui, pendant un temps et un temps seulement et bien partiellement d'ailleurs, ont été considérés comme la responsabilité de l'État. Les femmes dans le besoin n'ont jamais eu vraiment d'autre choix que de compter sur les groupes de base, d'entraide et de solidarité. Elles participent à des organisations, soit pour combler les besoins de base et assurer tant bien que mal leur survie (elles sont usagères des services), soit pour accomplir leur "devoir de citoyennes " et retourner à la communauté ce qu'elles ont reçu d'elle (elles sont travailleuses volontaires).

Dans la section précédente, il a été dit que, sans la présence de militantes et de bénévoles, bien des groupes de femmes auraient eu du mal à assurer leur fonctionnement et à s'installer dans la durée. Mais en dépit de son importance, le temps volontaire occupe un espace indéterminé à l'intérieur des groupes de base féministes et communautaires. Les motifs de l'engagement envers ses semblables et envers sa communauté sont multiples et évoluent selon les contextes et le type d'organisation. Les militantes ou les bénévoles viennent tantôt explorer, approfondir et partager des connaissances sur la santé, la violence, l'éducation populaire, tantôt 
"Si le "militantisme» et le "bénévolat" supposent tous deux un engagement social, le premier s'effectue en fonction d'une cause politique, voire idéologique alors que le second adopte une connotation morale, de charité et de dévouement." siéger sur différents comités afin d'assurer la représentation d'un segment de la communauté. Elles s'impliquent aussi parce que c'est là une manière de sortir de l'isolement et de chercher de l'aide. Un certain nombre de femmes qui effectuent du temps volontaire dans les groupes de base sont assistées sociales et leur niveau de participation indique qu'elles ne sont ni plus, ni moins engagées, ni plus, ni moins passives que la population en général. De ce fait, elles échappent à une définition essentialiste les décrivant comme des êtres misérables, désorganisés, dépendants et incapables de prise en charge ( St-Amand et Kérésit 1998; Michaud à paraître).

Majoritairement composés de femmes, tant dans la clientèle que dans le personnel, les groupes de base utilisent des modes d'organisation de leurs ressources humaines devant tenir compte de la participation plus ou moins importante de personnes pour qui les services rendus ne sont pas rétribués selon le mode du salariat. Si les intervenantes salariées ont en main une définition, somme toute claire, des tâches à accomplir, les volontaires, elles, sont souvent perçues comme des personnes ayant besoin de formation et d'encadrement. Si le « militantisme " et le "bénévolat » supposent tous deux un engagement social, le premier s'effectue en fonction d'une cause politique, voire idéologique alors que le second adopte une connotation morale, de charité et de dévouement. Le temps volontaire est fluide. Il va, il vient. Certains groupes ne comptent que sur celui-ci pour leur fonctionnement, d'autres vivent la décroissance du nombre de volontaires après avoir connu des années foisonnantes d'activités de toutes sortes organisées et maintenues par ces mêmes personnes. En raison sans doute de cette fluidité, le temps volontaire est devenu particulièrement controversé depuis que les gouvernements provinciaux ont adopté des politiques de workfare dont les points d'appui sont situés au coeur même du secteur communautaire.

Car le temps volontaire est un terme générique qui devrait, en principe, s'opposer à celui du workfare (Browne 1996 : 38). Mais depuis les modifications apportées au régime de citoyenneté sociale au Canada, le temps volontaire se superpose, pour ne pas dire qu'il se télescope au travail obligatoire. La perversion du temps volontaire au profit du travail obligatoire est inscrite à l'intérieur 
"En utilisant le chemin du temps volontaire, le travail obligatoire pénètre par la porte de service à l'intérieur des organisations communautaires et des groupes de femmes, laissant croire que les intervenantes des groupes ont encore les moyens politiques d'opposer une fin de non-recevoir à l'État néolibéral." de ce genre de programmes, au point que les décideurs et les gestionnaires n'ont pas besoin d'attendre que les groupes de femmes et les organismes communautaires se prononcent sur l'introduction de placements dans leurs structures organisationnelles. En utilisant le chemin du temps volontaire, le travail obligatoire pénètre par la porte de service à l'intérieur des organisations communautaires et des groupes de femmes, laissant croire que les intervenantes des groupes ont encore les moyens politiques d'opposer une fin de non-recevoir à l'État néolibéral.

Pour comprendre ce genre de perversion introduite par la politique-perversion de ce qui au départ est inscrit dans les principes et les modes d'intervention féministe et communautaire-il est nécessaire de rappeler les enjeux et les mandats que se donnent les groupes de base dont l'une des tâches essentielles et incontournables est de représenter les femmes auprès des institutions. Procédons d'abord à un rappel des enjeux. La violence est sans aucun doute un des thèmes qui a le plus structuré le mouvement des femmes en Ontario, au point que le réseautage des organisations qui interviennent dans ce domaine fait l'envie de bien d'autres secteurs de ce mouvement ${ }^{4}$. Mais cette grande visibilité de la violence ne doit pas nous faire oublier que d'autres enjeux structurent et fondent le caractère de ce dernier en Ontario français. Plusieurs organisations approchent la question des femmes de façon globale et holistique (Cardinal 1992; Coderre 1995; Welch 1999). À travers leurs activités, elles s'intéressent à l'éducation, à la santé, à l'autonomie sous toutes ses formes, à l'égalité juridique, à l'indépendance économique, au travail rémunéré et non rémunéré, au développement économique communautaire, au parrainage des femmes immigrantes, au racisme, à l'accès à la citoyenneté, à l'exploitation sexuelle des femmes et des enfants. Inutile d'allonger la liste car des oublis surviendraient forcément. Bien sûr, la pauvreté fait partie du nombre des enjeux mais ce ne sont pas tous les groupes qui en ont fait leur préoccupation principale. Chez la plupart d'entre eux, la pauvreté ne connait pas d'espace autonome sur lequel s'établit une plate-forme d'orientation politique. Car c'est souvent en travaillant sur d'autres besoins et surtout à travers leurs pratiques quotidiennes que les 
groupes de femmes ont été amenés à se pencher sur les problèmes aigus de pauvreté, obligeant l'action collective à se positionner par rapport à celle-ci.

Les intervenantes se voient reconnaitre la pauvreté en ce qu'elle émerge dans leur organisation comme la condition première d'un pourcentage important d'usagères des services, de militantes ou de bénévoles. La pauvreté émerge de bien des manières et rencontre des discours et des images multiformes de l'assistée sociale déjà en formation. Parfois, l'assistée sociale épouse les traits de la "nécessiteuse " qui vit avec des prestations tronquées de $21,6 \%$, qui se voit forcée par le système à reprendre contact avec un ex-conjoint violent pour obtenir des pensions alimentaires, qui est obligée de faire entre 20 et 40 demandes d'emploi par mois ou de se trouver un placement de workfare, qui vient de recevoir une lettre d'éviction de son logis et demande une place dans une maison d'hébergement.

Mais il arrive aussi que l'assistée sociale agisse comme militante ou bénévole avant d'être nécessiteuse. Alors, elle siège sur des comités et assure la représentation d'une partie de sa communauté; elle est cette voix qui, dans la nuit, répond à une ligne de crise; elle est celle qui collecte des fonds pour l'UNICEF, pour la banque alimentaire, pour la coopérative de vêtements usagés. Elle ira même jusqu'à fonder avec d'autres, une organisation qui lui ressemble, dans son identité et dans ses besoins (St-Amand et Kérisit 1998).

"Elle oblige aussi à repenser le regard que l'on pose sur elle pour $y$ opposer une image plus nuancée, $y$ compris $d u$ côté des groupes de femmes, dans la mesure où il existe des attentes contradictoires de la part des assistées et des volontaires d'une part, et des groupes de femmes d'autre part. " La plupart du temps, les groupes parviennent à la reconnaitre, pour peu que l'assistée sociale s'identifie comme telle-ce qui n'est pas toujours le cas-et pour peu aussi que le groupe ait développé une sensibilité à cet effet-ce qui ne va pas de soi non plus tant il est facile de regarder ailleurs et de ne pas poser de questions. L'assistée qui se dévoue et se démène oblige à réviser sa présence dans le contexte des réformes actuelles. Elle oblige aussi à repenser le regard que l'on pose sur elle pour y opposer une image plus nuancée, y compris du côté des groupes de femmes, dans la mesure où il existe des attentes contradictoires de la part des assistées et des volontaires d'une part, et des groupes de femmes d'autre part. D'un côté les assistées, au même titre que les volontaires, s'attendent à ce que leur travail soit reconnu dans 
"Les études historiques sur le sujet, comme celle de Margaret Little, nous indiquent que la régulation morale des mères seules est d'un degré bien plus élevé que lorsqu'il s'agit des pères..." l'éventualité d'une ouverture de poste qui leur donnerait le statut de salariée. Mais d'un autre côté, les ressources des groupes de femmes et des organismes communautaires continuent d'être rares et il est évident que les groupes ne peuvent satisfaire et les assistées et les volontaires au chapitre de l'emploi (Entrevue no. 2), c'est-à-dire permanent, bien rémunéré, par opposition à précaire et temporaire. Comme si la plupart des intervenantes ne travaillaient pas elles-mêmes dans des conditions précaires d'emploi et de salaire.

Quoi qu'il en soit, les situations de pauvreté depuis 1995 ont forcé les groupes de femmes et les organismes communautaires à élargir leur mandat d'origine. Certaines maisons d'hébergement par exemple, face à la réalité des femmes menacées d'éviction de leur logis, ont pris le parti d'élargir la définition de la violence pour inscrire celle de la rue et celle des institutions (Entrevues nos. 10-11). Nombre de groupes, et ceci vaut pour presque tous ceux qui ont participé à cette recherche, sont amenés à représenter les femmes confrontées à de multiples situations : ouvrir un compte de banque, faire une demande d'aide sociale, se présenter à la cour pour la pension alimentaire, se défendre dans une cause criminelle ou en appeler d'une décision arbitraire (Entrevues nos. 3-7-8-10-11-14). Représenter ici les femmes veut dire les soutenir, parler pour elles dans leurs démarches auprès des institutions (Spivak 1988).Dans toutes les situations, ce type de représentation est tributaire des images qui circulent à propos de l'assistée sociale. Les études historiques sur le sujet, comme celle de Margaret Little, nous indiquent que la régulation morale des mères seules est d'un degré bien plus élevé que lorsqu'il s'agit des pères (1998:153). Cette régulation morale n'est pas que purement symbolique. Elle prend forme dans les pratiques quotidiennes des agents d'aide sociale qui appliquent des règles qui, dans certains cas, menacent la sécurité des femmes et de leurs enfants. Par exemple, lorsque le système exige qu'elles reprennent contact avec un ex-conjoint violent afin d'obtenir des allocations familiales ${ }^{5}$.

Les préjugés, la discrimination et l'exclusion ciblent l'ensemble des assistés mais de façon différente selon le sexe, l'ethnicité, les arrangements familiaux, l'âge, l'appartenance linguistique. Si les 
"Le discours féministe à propos de l'assistée - en partie façonné par sa présence et son action au sein des groupes-n'est cependant ni uniforme ni exempt d'ambiguité. Il offre l'image réfractée d'un prisme plutôt qu'une image fixe. " intervenantes - devenues accompagnatrices, traductrices, représentantes juridiques — sont souvent témoins des situations de danger, du mépris et des préjugés auxquels fait face l'ensemble des assistés, elles croient que leur présence augmentera les chances d'une décision favorable. Le discours féministe à propos de l'assistéeen partie façonné par sa présence et son action au sein des groupes—n'est cependant ni uniforme ni exempt d'ambiguïté. Il offre l'image réfractée d'un prisme plutôt qu'une image fixe. Si du côté des intervenantes féministes et progressistes du secteur communautaire, nous ne devons pas nous attendre à retrouver les mêmes préjugés, le même mépris, les mêmes attitudes de discrimination qui foisonnent ailleurs dans d'autres secteurs de la communauté et des institutions, il n'est pas acquis par contre que ces mêmes groupes songent à accorder à l'assistée sociale une certaine forme d'autonomie d'action et de parole. C'est donc dire que les discours sur l'opprimée ou sur l'individu autonome déterminent les représentations et les positions que les groupes prennent envers elle et en son nom.

Or, il n'y a pas que la représentation de l'expérience vécue par l'assistée sociale qui intervient dans la formation du discours et des pratiques féministes. Il y a aussi la représentation du groupe lui-même; la nécessité pour l'organisation de se faire entendre, de se faire comprendre, de se faire reconnaitre comme force sociale légitime.Y aurait-il ici une sorte de concurrence entre les entitésentre femmes et organisations-qu'il s'agit de représenter? Non, car il ne s'agit pas à proprement parler d'une sorte de substitution de l'expérience vécue au profit de la mise en valeur de l'expertise organisationnelle vis-à-vis la communauté des femmes que les groupes desservent. Le discours des groupes, féministes et progressistes, est constitué de la présence constante des catégories de sujets auxquels ils s'adressent et leurs pratiques s'articulent autour des divers systèmes d'oppression. Mais ces mêmes groupes de base font constamment valoir leur situation privilégiée, que nulle instance institutionnelle ni gouvernementale ne saurait occuper à leur place. Leur présence est incontournable, ce qui leur permet de revendiquer, à la fois, reconnaissance et financement. 
Ensuite, survient le dilemme de choisir entre la représentation de l'assistée-victime d'oppression et celle de l'assistée sociale politiquement autonome, capable de parole et d'action, réduisant ainsi, de façon certaine, l'expérience vécue. Prenons à titre d'exemple ce propos d'une intervenante dont l'organisation est en train de développer une stratégie permettant à certaines femmes d'échapper au contrôle du système :

"Nous sommes en train de développer de l'expertise pour obtenir, pour nos clientes, des pensions d'invalidité, qui est la seule façon de s'en sortir. Les médecins sont noyés de demandes apparemment. J'ai des clientes où la situation précaire [...] dont la santé mentale s'est détériorée à cause de tout ça. Et donc, elles ne sont plus vraiment, en ce moment, dans un état de penser qu'elles pourraient travailler ou suivre des cours. Donc, elles ont droit, elles se qualifient pour l'invalidité » (Entrevue no. 11).

"L'assistée peut être tout à la fois victime et autonome, c'est-à-dire qu'elle peut épouser les traits de la "nécessiteuse " qui vit avec des prestations tronquées de 21,6\% en même temps que ceux de la militante ou de la bénévole qui agit au-delà et en dépit de la misère. Le danger serait de chercher à séparer ces deux réalités..."
À court terme, cette approche a le mérite d'accorder un répit à des femmes menacées de perdre l'unique revenu qui leur reste. Mais les effets à long terme d'un tel diagnostic ne risquent-ils pas d'ancrer perpétuellement les assistées sociales dans un statut de victime ? Ne risquent-elles pas d'être soumises à un autre contrôle tout aussi contraignant que l'aide sociale, celui de la médecine psychiatrique, consolidant une série d'images et de perceptions négatives à propos des femmes ?

L'assistée peut être tout à la fois victime et autonome, c'est-àdire qu'elle peut épouser les traits de la "nécessiteuse " qui vit avec des prestations tronquées de $21,6 \%$ en même temps que ceux de la militante ou de la bénévole qui agit au-delà et en dépit de la misère. Le danger serait de chercher à séparer ces deux réalités : de traiter uniquement avec la victime sans considérer la partie autonome qui motive son agir. L'implication des femmes assistées sociales au sein des organisations interdit la description étroite et essentialiste de la victime de pauvreté qui a du mal à prendre des décisions par elle-même, de s'organiser, de lutter contre les situations d'oppression qui l'assaillent quotidiennement. 


\section{La position des groupes de femmes vis-à-vis les placements de workfare}

"Il n'est pas rare de voir qu'une position décidée au niveau d'un regroupement ou d'une coalition de solidarité soit nuancée, même contredite au niveau local. $\mathrm{La}$ question du travail obligatoire offre un bon exemple de ce type de décalage. "
L'énoncé affirmant que chaque groupe soit implanté dans son milieu ne veut rien dire en soi s'il n'est pas accompagné d'un autre, portant sur la formation et la dynamique des réseaux d'échange. Chaque entité du secteur communautaire envisage la possibilité de se lier à d'autres groupes avec des orientations plus ou moins similaires. Comme un bon nombre de ces échanges sont conjoncturels, il est plus facile de nouer des liens entre organisations dont les objectifs et les orientations ne sont pas forcément les mêmes. La géographie des réseaux est fort complexe et ne peut être perçue que sur un seul plan. Certes, les groupes se rassemblent d'abord sur la base d'enjeux similaires sur lesquels ils travaillent, à l'instar d'Action ontarienne, regroupement autour de la violence faite aux femmes. Puis, au-delà des enjeux qui les rassemblent, les groupes se concertent parce qu'ils partagent la cause des femmes, sans nécessairement partager la même orientation féministe. C'est le cas de la Table féministe de concertation provinciale qui, en créant cette structure "parapluie ", forme une coalition de solidarité de premier niveau. De plus, ces échanges débordent souvent la scène d'un même mouvement pour rejoindre, à d'autres niveaux, d'autres mouvements sociaux. Les groupes rencontrés ne sont pas tous rattachés directement à la Table féministe de concertation provinciale. La très grande majorité par contre, relève d'un regroupement provincial lequel, parfois, mais pas nécessairement, fait partie de cette importante coalition de solidarité féministe en Ontario français.

La géographie des réseaux, avec ses regroupements locaux et régionaux et au-delà, celle de l'Ontario francophone et anglophone, ne doit pas nous faire oublier que chacune des entités concernées préserve son autonomie. Il n'est pas rare de voir qu'une position décidée au niveau d'un regroupement ou d'une coalition de solidarité soit nuancée, même contredite au niveau local. La question du travail obligatoire offre un bon exemple de ce type de décalage. 
"en y regardant de plus près, on constate que la décision d'accepter ou de refuser des placements de travail obligatoire tient moins de la solidarité des coalitions et des regroupements, et de leurs positions politiques que de la relation développée avec l'assistée sociale. "
La position qui domine à l'heure actuelle dans le secteur communautaire est celle du refus des placements de travail obligatoire. Les groupes de femmes, les organismes communautaires sont quasi unanimes à le condamner. Cela va de soi semble-t-il. Du côté des intervenantes féministes, on ne semble pas s'être enlisées dans d'interminables débats sur les méfaits d'un tel programme qui cible les organisations autant que les assistés sociaux. On pourrait même se demander si le débat a bel et bien eu lieu. Bon nombre de mes répondantes ont précisé que la position de leur groupe n'avait suscité qu'une brève discussion au sein de leur conseil d'administration et que dans les faits, la décision de se prononcer contre des placements de travail obligatoire découlait de la Table féministe de concertation provinciale, confirmant l'importance du leadership de cette coalition ${ }^{6}$.

Mais, en y regardant de plus près, on constate que la décision d'accepter ou de refuser des placements de travail obligatoire tient moins de la solidarité des coalitions et des regroupements, et de leurs positions politiques que de la relation développée avec l'assistée sociale. À quelques reprises, j'ai rencontré des intervenantes locales ou régionales qui avaient adopté une position différente de celle de leur regroupement. L'instant crucial se joue lorsque les femmes-forcées de se trouver un placement de workfare-se présentent à la porte des groupes. Les femmes approcheront alors d'autant plus facilement les organisations qui les connaissent. Les motifs et justifications pour accepter de telles demandes sont multiples et pas nécessairement clairs dans l'esprit des répondantes féministes. On pourra invoquer, par exemple, que ce ne sont pas réellement des placements dans la mesure où l'assistée concernée fait déjà du temps volontaire au sein de l'organisation. Cette confirmation survient après qu'on ait pris soin d'affirmer la position du groupe contre le workfare. C'est donc dire qu'on peut à l'occasion faire une exception pour les assistées de l'intérieur-militantes ou bénévoles—celles dont on connait la situation et pour qui l'insoutenable réalité de se faire couper ses maigres prestations ne saurait venir du groupe luimême. L'intention ici est honnête et ne se veut pas en contradiction avec la position de l'organisation qui demeure farouchement 
opposée à l'implantation du workfare. Mais la complication survient lorsque le système d'aide sociale prend la situation au sérieux et entend l'inscrire formellement dans ses dossiers. Dans ce cas, le groupe pourra réaffirmer sa position de refus, c'est-à-dire rejeter toute possibilité de placement, y compris pour celles qui font du temps volontaire, afin de demeurer en accord avec ses convictions comme l'indique l'extrait suivant.

"On n'a pas dit qui, mais c'était une femme qui était active. Et c'est là qu'on a décidé qu'il ne fallait pas prendre et analyser cette demande, la personnaliser. Parce que là, les gens voulaient savoir qui, qui, qui. On a dit non. Parce qu'il ne faut pas prendre une décision selon la personne qui en fait la demande. Et c'est là qu'on a dit : "Bien non. C'est malheureux, mais on refuse". Et on doit répondre [...]. "Oui, elle participe. Elle est active. Elle a un rôle très important. Elle contribue beaucoup à notre groupe. Mais non, ça ne doit pas être perçu comme un placement et on trouve que c'est tout à fait inacceptable" "(Entrevue no. 13).

"Les groupes locaux sont au fait des positions prises par leur regroupement au niveau provincial mais les répondantes de ces groupes vont surtout insister sur leur position de témoins privilégiés de la détresse extrême qui guette les assistées advenant l'arrêt, ne serait-ce que temporaire, des prestations. "
D'autres groupes féministes ou communautaires vont par contre s'appuyer sur la dure réalité de l'assistée sociale, son état de santé, physique et mentale, sa situation de misère et d'indigence. Les groupes locaux sont au fait des positions prises par leur regroupement au niveau provincial mais les répondantes de ces groupes vont surtout insister sur leur position de témoins privilégiés de la détresse extrême qui guette les assistées advenant l'arrêt, ne serait-ce que temporaire, des prestations. À la position de refus catégorique qui souligne le côté pervers du workfare se superpose une position présentée comme étant conforme à la réalité. Du côté de ces intervenantes, on dit très bien comprendre l'analyse politique qui a prédominance dans le secteur communautaire. Mais cette analyse n'apporte pas de solution immédiate aux difficultés particulières des assistées. Dans ce cas-là, l'acceptation des placements de workfare pourra éventuellement s'étendre au-delà de celles qui font du temps volontaire pour s'ouvrir comme possibilité à d'autres assistées sociales du milieu. 
"...le rôle du groupe n'est pas de faire la police pour le système d'aide sociale, ni d'offrir des placements qui seraient inutiles, surnuméraires aux activités de l'organisation."
"Ici, nous avons eu plusieurs femmes qui font des placements. Je ne suis pas contre personnellement. Je suis contre un travail forcé. Mais l'idée de les mettre dans des organisations pour apprendre, je ne suis pas contre ça parce que j'ai eu beaucoup de succès ici. Je sais que c'est forcé et que c'est attaché à l'argent. C'est la philosophie. Tout le monde a de la misère avec ça. Si ça n'était pas forcé, si ça serait comme avant avec Boulot Ontario qui était la même chose. Moi je pense que c'était une bonne chose. [...] Alors, on avait une bénévole qui faisait ça à tous les jours. Elle était sur le bien-être social et puis c'est elle qui a fait la demande. Elle a dit: "Est-ce que je peux embarquer sur le programme, ça me donnerait un petit peu plus d'argent?" ". Elle a mis ça sur son CV.Au lieu d'être juste bénévole, elle peut dire qu'elle était employée. Elle a fait ça pendant six mois. On l'a renouvelée et ensuite elle s'est trouvé un emploi à temps plein" (Entrevue no. 3).

Un autre motif d'acceptation d'un placement touche la protection des assistées sociales contre des situations abusives qui pourraient se présenter avec des employeurs sans scrupule, tentés de profiter de leur vulnérabilité (Entrevue no. 9). À cela, on ajoute que le rôle du groupe n'est pas de faire la police pour le système d'aide sociale, ni d'offrir des placements qui seraient inutiles, surnuméraires aux activités de l'organisation.

"J'ai été très claire, je peux te dire. Que jamais que je m'aperçoive qu'il y a de l'abus_-parce que ça ne sera pas drôle -ils vont être dénoncés quelque chose de rare. Jamais, jamais—c'est moi qui suis la coordonnatricejamais que j'accepterai que "Ontario au travail " me dise : "tu vas faire ci et tu vas faire ça et tu vas leur faire faire ci et th vas leur faire faire ça». Pantoute! Jamais! Puis c'est ça que j'ai expliqué à [nom de l'assistée]. [...]. Mais je lui ai dit que si elle veut être réceptionniste, si c'est ça qu'elle veut apprendre, ça va 
"Qu'il $y$ ait eu refus ou acceptation plus ou moins limitée des placements de workfare, il ne semble pas $y$ avoir eu de pression ou d'intimidation. Cela s'est fait de plein gré et en toute connaissance de cause et, dans la plupart des cas, sans qu'il y ait eu d'entente tacite avec le système d'aide sociale. " être son choix. Le travail qu'elle va faire, ça va être son choix. Pourvu qu'elle réponde au téléphone comme il faut [...]. Après avoir dit ce que j'avais à dire, que je voulais en aucune considération entendre dire qu'il $y$ avait de l'abus ou quoi que ce soit, ils ont tous été d'accord avec moi. Tous. Nous avons un docteur, un pharmacien, deux infirmières, un psychiatre, un psychologue pour le groupe. Nous avons aussi un avocat. Tous les membres du conseil ont signé pour le respect de la confidentialité et le respect de la personne. Tout le monde a signé »(Entrevue no. 9).

Qu'il y ait eu refus ou acceptation plus ou moins limitée des placements de workfare, il ne semble pas y avoir eu de pression ou d'intimidation. Cela s'est fait de plein gré et en toute connaissance de cause et, dans la plupart des cas, sans qu'il y ait eu d'entente tacite avec le système d'aide sociale. Cependant, une telle entente est possible et selon les intervenantes concernées, résulte de négociations sur les modalités, à la satisfaction, semblet-il, du groupe lui-même, comme l'indique l'échange qui suit.

$\mathbf{R}$ : Avec l'aide sociale, voilà. Donc, la municipalité, comment ça fonctionnait? Par exemple, quelqu'un veut faire un placement communautaire. La personne vient nous voir et nous dit : "Bien écoutez, j'aimerais faire un placement communautaire ". Nous, on dit: "Bon, tu veux faire un placement "? On écrit à sa travailleuse comme quoi on l'a acceptée. Voilà sa description de tâches. Voilà les horaires. La personne va rencontrer sa travailleuse. La travailleuse lui fait remplir une fiche de consentement, faxe ça aux services de placement. Les services de placement avec lesquels nous travaillons étroitement, les services de placement de la municipalité viennent nous rencontrer. On avait déjà négocié les fonds avec eux. Et puis à un moment donné, eux, ils avaient même pensé à faire un transfert de fonds. Tu vois, ça été quelque chose qui avait été discuté avant moi pour que nous, on puisse... Et puis nous, on a refusé. On a dit : "Non, on ne veut pas avoir les gens sur le dos. Faites ça vous-mêmes ". Donc, c'est extrêmement intéressant.

Q. Donc, autrement dit, vous avez un salaire de plus, en terme de financement qui leur vient d'eux, en recevant des placements. 
R. Bien oui parce que finalement...

Q. Puis les femmes, elles, elles ont aussi un $250 \$$ de...

I. C'est pas un salaire de plus en tant que tel. C'est par exemple, quand tu as $250 \$$, plus tes frais de garderie qui sont complètement pris en charge, plus ton transport, tu es bien.

Q. Bien sûr.

R. Et c'est ça qui a changé. Les femmes ont commencé à sortir en masse de chez elles, en masse. En plus, ils nous envoient $100 \$$ par participante, ce qui nous permet de leur donner des services directs. Par exemple, on dit à l'une d'elles: " Bon bien toi, c'est toi qui vas recevoir. Donc, tu vas t'occuper des autres».Tu vois?

Q. Et combien de temps dure le placement? Onze mois?

R. Onze mois par projet.

Q. Par projet, par personne.

R. Et par personne.

Q. Voilà.

R. Ou six mois. Ça dépend. Six mois quand c'est des. parce qu'il $y$ a deux types de placements. Il y a le placement dans le cadre d'un projet et il y a les placements quand tu crées une position. Par exemple, tu as besoin de... Mais je n'aime pas ça tellement. Tu as besoin par exemple d'une secrétaire et tu vas leur dire : "Bien, j'ai besoin d'une secrétaire ». Mais je n'aime pas ça, moi. Je n'aime pas ça parce que je pense que c'est réellement du travail obligatoire. Donc, c'est surtout dans le cadre de projets, c'est plus intéressant parce que les femmes discutent entre elles, travaillent sur le projet ensemble et puis se donnent une orientation. Et puis au bout de trois, quatre mois de placements, souvent, on a placé la

"Présentement, il est très difficile d'avoir l'heure juste quant au nombre de groupes de base qui participent plus ou moins formellement au programme Ontario au travail." " personne en milieu de travail. La personne a déjà trouvé un emploi (Entrevue no. 17).

Présentement, il est très difficile d'avoir l'heure juste quant au nombre de groupes de base qui participent plus ou moins formellement au programme Ontario au travail. Le nombre de ceux qui ont un protocole d'entente dûment signé avec les services sociaux de leur région est déjà très restreint. Par exemple, en mai 1998, selon un document de la Table féministe de concertation 
"Ce communiqué est silencieux sur un ensemble d'éléments essentiels qui nous permettraient d'évaluer la profondeur de cette soi-disant réussite..." provinciale, sur 1,220 organismes contactés dans la région d'Ottawa-Carleton, seulement 101 ont accepté de participer, soit moins de $10 \%{ }^{7}$. Mais en juin dernier, le ministère des Services sociaux et communautaires a émis un communiqué vantant la réussite du programme. Sur 43 régions, 27 sont parvenues à dépasser le minimum requis par la province, au-delà duquel une augmentation des subventions est prévue. Parmi les régions qui sont parvenues à un tel exploit, on retrouve Toronto et Sudbury. Mais parmi les régions qui n'ont pas atteint l'objectif fixé par le ministère, il faut nommer Thunder Bay, Prescott-Russell et Ottawa-Carleton (Ministry of Community and Social Services 2000). Ce communiqué est silencieux sur un ensemble d'éléments essentiels qui nous permettraient d'évaluer la profondeur de cette soi-disant réussite : les coûts associés à la gestion du programme, les méthodes des calculs des régions, le nombre de placements communautaires par rapport au secteur privé, le nombre de protocoles d'entente dûment signés entre les demandeurs de placements et le programme régional d'Ontario au travail, le genre de postes comblés par rapport aux expertises disponibles.

\section{Conclusion}

Le temps volontaire est effectué par plusieurs catégories d'individus, parfois, mais pas nécessairement, en demande de reconnaissance et d'emploi permanent.Toutes sortes de catégories sociales de femmes font du bénévolat : des militantes, des femmes de toutes les origines et de toutes les fortunes. Parmi celles qui donnent du temps volontairement, il y a des assistées sociales qui, parfois, s'impliquent dans plus d'un organisme. L'enquête faite par le Conseil de planification sociale d'Ottawa-Carleton révèle que "parmi les 68 répondants et répondantes, $54 \%$ rapportent qu'ils avaient travaillé bénévolement dans un organisme communautaire avant de participer au programme Ontario au travail (1999 : 20). Dans le contexte actuel, une des étapes importantes dans le travail des groupes de femmes et des 
"Persister à y voir des contradictions signifie qu'on n'a pas vraiment saisi le caractère situationnel de l'assistée sociale. Ce qui revient à lui accorder une image fixe qui ne bouge pas, une image de presque victime qui n'a de parole que lorsque sa représentation passe par les groupes qui entendent la défendre et représenter ses besoins. " organisations communautaires serait de réfléchir aux motifs de ce temps volontaire. Nous avons vu précédemment comment les assistées sociales sont capables de remplir plusieurs fonctions. Cela veut dire aussi qu'elles peuvent circuler entre des situations qui semblent à première vue contradictoires, comme par exemple faire une requête de travail obligatoire dans un groupe quelconque à midi, et s'opposer farouchement, dans la même journée, à ce que le Conseil d'administration d'un autre organisme accepte des placements de workfare sous le prétexte que ça ferait du bien aux assistés sociaux de travailler un peu. Persister à y voir des contradictions signifie qu'on n'a pas vraiment saisi le caractère situationnel de l'assistée sociale. Ce qui revient à lui accorder une image fixe qui ne bouge pas, une image de presque victime qui n'a de parole que lorsque sa représentation passe par les groupes qui entendent la défendre et représenter ses besoins. De cette première réflexion découle une seconde requérant l'évaluation des besoins spécifiques des assistées sociales non seulement sur une base individuelle - comme l'approche du cas par cas, parfois nécessaire mais typique d'une intervention faite en fonction de la demande des usagères de services - mais aussi collectivement. Estce que l'ensemble des assistés sociaux-femmes ou hommessera en meilleure position pour rompre le cercle vicieux de la pauvreté avec le programme Ontario au travail? Peuvent-ils ou peuvent-elles espérer autre chose que des placements répétitifs de six mois? Et pour combien de temps? L'organisation est-elle en position d'offrir un poste à temps plein à une personne en demande de placement? Et en quoi quelques cas de réussite peuvent-ils compenser tant de déceptions?

Les groupes doivent se prémunir contre un des effets pervers de la politique : celui de limiter, voire détourner, le temps volontaire au profit du travail obligatoire. Le fait que la majorité des organismes qui composent ce secteur ne semble pas vouloir s'aligner avec les visées du programme ne change rien au fait que le temps volontaire puisse être exploité un peu à la manière d'une ressource naturelle. Si, de façon brutale et autoritaire, le gouvernement ontarien s'est efforcé, depuis 1995, à démanteler les canaux de concertation et de communication entre l'État et la 
"...les groupes risquent de se voir reconduits dans leur statut d'intermédiaires, plus que jamais coincés entre la nécessité et l'État néolibéral, par une voie qu'ils n'ont pas cherchée. » société civile afin, soi-disant, de se soustraire aux pressions des groupes d'intérêts, de manière implicite cependant, les groupes risquent de se voir reconduits dans leur statut d'intermédiaires, plus que jamais coincés entre la nécessité et l'État néolibéral, par une voie qu'ils n'ont pas cherchée. Les tendances à la segmentation des sans-emploi font en sorte que les catégories sociales les plus exclues du travail salarié sont " assignées à résidence » à l'intérieur d'un secteur communautaire à qui l'État impose le «statut de sous-traitant » (Browne 1999 : 47).

Aussi, les groupes doivent-ils se soumettre à une réflexion en profondeur sur l'évolution du travail d'intervention des dernières années. Les travailleuses impliquées dans l'intervention féministe et communautaire sont aussi des intellectuelles qui prêtent forme à des discours sur la pauvreté des femmes, des intellectuelles qui attribuent un sens et une justification à un ensemble de pratiques discursives à propos de l'assistée sociale et qui interprètent quotidiennement ses besoins et ses désirs-ce qui en bout de ligne pourrait bien être l'interprétation de leurs propres besoins et leurs propres désirs (Spivak 1988). Un tel travail de réflexion, il va sans dire, nécessite le renforcement du réseautage au niveau provincial entre groupes de base et entre mouvements sociaux intéressés à poursuivre un plan d'action efficace contre tout un ensemble de politiques qui, à l'instar du workfare, vise à faire pression sur les salaires et sur la déqualification de certaines catégories d'emplois en Ontario.

\section{Bibliographie}

ANDREW, Caroline (1998). "Les femmes et l'État-providence : question revue et corrigée ", Politiques et sociétés, vol. 17, nos, 1-2,171-182.

BRISKIN, Linda and MONA, Eliasson, (1999). «Preface : Collaboration and Comparaison », Linda BRISKIN and Mona ELIASSON (eds) Women's Organizing and Public Policy in Canada and Sweden, Montreal \& Kingston - London - Ithaca : McGill-Queen's University Press, vii-xxi. BROWNE, Paul Leduc (1999). "La dialectique de l'économie sociale : travail, employabilité, solidarité », Reflets, vol. 5, no. 1, 28-53.

BROWNE, Paul Leduc (1996). Love in a Cold World ? The Volontary Sector in an Age of Cuts, Ottawa : Canadian Centre for Policy Alternatives.

CHOLETTE, Chantal (1998). «Le travail obligatoire en Ontario : solution ou imposture? », Reflets, 
vol. 4 , no 1 .

CHOLETTE, Chantal (1997). Inventaire des politiques néolibérales adoptées par le gouvernement de l'Ontario au cours de la méga-semaine et de certaines recommandations de la Commission de révision des formalités administratives, préparé pour la Table féministe de concertation provinciale de l'Ontario. 46 pages.

CARDINAL, Linda (1992). « La recherche sur les femmes francophones vivant en milieu minoritaire : un questionnement sur le féminisme ", Recherches féministes, vol. 5, no. 1, 5-29.

CODERRE, Cécile ( 1995). "Femmes et santé en français s'il-vous-plaît ", Reflets, vol. 1, no. 2, 39-71.

DANDURAND, Renée B. et McALL, Christopher (1996). "Welfare, workfare, wedfare : faut-il encore assister les mères seules? ", Lien social et politiques - RIAC, vol. 36, 79-91.

ÉQUIPE DE TRAVAIL SUR LE SUIVI DU PROGRAMME ONTARIO AUTRAVAIL (1999). Dire les choses telles qu'elles sont : Espoir et réalité, Ottawa : Conseil de planification sociale d'Ottawa-Carleton, 36 pages.

FELDBERG, Georgina and CARLSSON, Marianne (1999). «Organized for Health :Women's Activism in Canada and Sweden ", dans Women's Organizing and Public Policy in Canada and Sweden, Montreal \& Kingston - London and Ithaca : McGill-Queen's University Press, 347-374.

JENSON, Jane and PHILLIPS, Susan D. (1996). "Regime Shift : New Citizenship Practices in Canada ", International Journal of Canadian Studies, no. 14, 111- 135.

JENSON, Jane (1997). «Fated to Live in InterestingTimes :Canada’s Changing Citizenship Regimes », Revue canadienne de science politique, vol. 30, no. 4, 627-644.

LAURIN, Nicole (1999). « Le démantèlement des institutions intermédiaires de la régulation sociale : vers une nouvelle forme de domination ", Sociologie et sociétés, vol. 31, no. 2, 65-72.

LAMOUREUX, Jocelyne (1994). Le partenariat à l'épreuve, Montréal, Les éditions Saint-Martin.

LITTLE, Margaret Jane Hillyard (1998). 'No Car, No Radio, No Liquor Permit' : The Moral Regulation of Single Mothers in Ontario, 1920-1997, Toronto, Oxford, New York, Oxford University Press.

LUSTIGIER-THALER, Henri, MAHEU, Louis et HAMEL, Pierre (1998). « Enjeux institutionnels et action collective ", Sociologie et sociétés, vol. XXX, no. 1,173-187.

MAHON, Rianne (1999). "Both Wage Earner and Mother " : Women's Organizing and Chilcare Policy in Sweden and Canada, dans Women's Organizing and Public Policy in Canada and Sweden, Montreal \& Kingston - London - Ithaca, McGill-Queen's University Press, 238-279. MICHAUD, Jacinthe (à paraître, automne 2000 ). «Stratégies de résistance, stratégies de contournement : l'auto-représentation des femmes francophones et le travail obligatoire en Ontario ", dans Andréa Martinez et Christine Olivier (dir.) La tension tradition-modernité chez trois groupes minoritaires de femmes en situation de vulnérabilité, Ottawa, Les Presses de l'Université d'Ottawa.

MICHAUD, Jacinthe (1997). "Le mouvement des Centres de santé : grandeur et misère de la participation et stratégies politiques de transformation du discours de l'État ", Nouvelles Pratiques Sociales, vol. 10, no. 1, 97-110.

MICHAUD, Jacinthe (1995). Angel Makers or Trouble Makers ? The Health Centres Movement in Québec and the Conditions of Formation of a Feminist Counter-Hegemony on Health, Ph.D dissertation, Toronto, University of Toronto. 
MICHAUD, Jacinthe (1992). «The Welfare State and the Problem of Counter-Hegemonic Responses Within the Women's Movement ", dans William K. Carroll (ed) Organizing Dissent : Contemporary Social Movements in Theory and in Practice, first edition. Toronto, Garamond Press, 200-214.

MINH-HA,Trinh T. (1989).Woman Native Other, Bloomington \& Indianapolis : Indiana University Press.

MOSCOVITCH, Allan (1997). "Social Assistance in the New Ontario », dans Diana Ralph, André Régimbald and Nérée St-Amand (eds), Mike Harris's Ontario : Open for Business, Closed to People, Halifax, Fernwood Publishing, 80-91.

ROCHE, Maurice (1995). "Rethinking Citizenship and Social Movements : Themes in Contemporary Sociology and Neoconservative Ideology ", dans Louis Maheu (ed) Social Movements and Social Classes :The future of Collective Action, London, Sage, 186-219. SPIVAK, Gayatri Chakravorty (1988). "Can the Subaltern Speak ", dans Cary Nelson and Lawrence Grossberg (eds) Marxism and the Interpretation of Culture, Urbana \& Chicago, University of Illinois, 271-313.

ST-AMAND, Nérée et KÉRÉSIT, Michèle (1998). Pauvreté et nouvelles solidarités : repenser l'intervention, Montréal, Éditions Saint-Martin.

WELCH, David (1999). "L'économie sociale en Ontario français : analyse historique, pratiques actuelles et recherche se sens ", Reflets, vol. 5, no. 1, 55-74.

WORKFARE WATCH (1999). Broken Promises : Welfare Reform in Ontario, Toronto :Workfare Watch c/o Community Social Planning Council of Toronto.

\section{Notes}

1. Chez les théoriciennes de la post-colonialité (Spivak 1988; Trinh T. Minh-ha1989 : 67-68), la notion de représentation possède deux significations lesquelles, en dépit de leur discontinuité, agissent concurremment. Il y a d'abord la représentation qui veut dire : « To speak for », «parler au nom de » la subalterne, (ici de l'assistée) et re-présentation au sens d'une description concrète de la réalité, de l'expérience vécue (Spivak 1988 : 275). C'est en m'inspirant de cette double signification que je vais aborder le travail de représentation fait par les groupes de femmes.

2. Je remercie un de mes participants de m'avoir confirmé ce fait à partir de ses analyses et de ses pratiques quotidiennes.

3. Ce règlement a été renversé par la Cour d'Appel de l'Ontario pour le motif qu'il était fondé sur des stéréotypes sexistes contraires à la Charte des droits et libertés.

4. Le Forum sur la santé des femmes qui a eu lieu à l'Université d'Ottawa en novembre 1999, mis sur pied par plusieurs organisations féministes, dont l'Union Culturelle des Franco-Ontariennes et la Table féministe de concertation provinciale, vise à promouvoir un réseau féministe en santé similaire à l'Action ontarienne contre la violence faite aux femmes. Les suites de ce Forum sont à venir.

5. Je fais ici référence à des entrevues de la première série avec les femmes francophones.

6. Cette coalition a organisé en 1998 une série de forums dans trois régions stratégiques de l'Ontario (Ottawa,Toronto, Sudbury) sur la réforme de l'aide sociale et le nouveau programme de workfare avec l'objectif de sensibiliser et d'informer les femmes et les groupes du secteur communautaire. 
7. Ces chiffres ont été compilés par Chantal Cholette pour le compte de la coalition. On y apprend, en outre, que seulement 89 placements ont été effectués dans cette région, entre novembre 1997 et avril 98 et qu'au cours de cette période, 17 personnes n'ont pu terminer le programme, soit que le placement ne convenait pas, soit qu'elles ne se sont pas présentées. 